\title{
Bloedverdunning anno 2016
}

\section{Nie} IN LEIDING
Niet lang geleden schreven artsen bij veneuze trombotische problemen (diepe veneuze trombose, longembolie, atriumfibrilleren) meestal een vitamine K-antagonist (VKA) voor en bij arteriële problemen (myocardinfarct, herseninfarct zonder atriumfibrilleren) aspirine. Intussen zijn er nieuwe antitrombotische middelen beschikbaar gekomen en soms heeft het combineren van middelen een meerwaarde. De laatste tijd krijgen we over dit thema steeds meer vragen van huisartsen. In dit artikel geven we een overzicht van de ontwikkelingen rond bloedverdunning anno 2016. Voor de weergave van

\begin{abstract}
Samenvatting
Willemsen R, Dinant GJ, Verheugt F, Ten Cate H, Weerkamp N. Bloedverdunning anno 2016. Huisarts Wet 2016;59(7):312-8.

Naar aanleiding van vragen van huisartsen vatten we in dit artikel de actuele stand van zaken rond antitrombotische medicatie samen. We gaan in op de pathofysiologie van de trombusvorming en behandelen de verschillende soorten antitrombotische medicamenten die beschikbaar zijn. Vervolgens bespreken we per indicatie welke middelen de voorkeur hebben. Pathologische trombusvorming kan onderverdeeld worden in veneuze trombose (zoals bij longembolieën) en arteriële trombose (zoals bij hartinfarcten en herseninfarcten zónder atriumfibrilleren). In beide gevallen is er in de regel een indicatie voor preventie met antitrombotische middelen. In orale vorm zijn er twee soorten antitrombotische medicamenten: de plaatjesremmers en de orale anticoagulantia $(O A C)$. Bij een verhoogd risico op arteriële trombose is er veelal een plaatjesremmer geïndiceerd. Dit is het geval bij stabiel kransslagaderlijden, een doorgemaakt herseninfarct en perifeer arterieel vaatlijden. Bij veneuze trombose of een risico daarop (zoals bij atriumfibrilleren, diepe veneuze trombose of longembolie) is er meestal een indicatie voor behandeling met een OAC. Per indicatie varieert de voorkeursbehandeling echter en soms is er plaats voor combinaties van verschillende antitrombotische medicamenten. Daarnaast moet men het bloedingsrisico per individuele patiënt meewegen, al vormt dat risico maar zelden een harde contra-indicatie voor antitrombotische medicatie. Combinaties van twee verschillende plaatjesremmers zijn in sommige situaties (tijdelijk) te prefereren boven monotherapie met één plaatjesremmer, bijvoorbeeld na een doorgemaakt myocardinfarct. Incidenteel kan men drie middelen combineren, bijvoorbeeld bij patiënten met een doorgemaakt myocardinfarct én atriumfibrilleren.
\end{abstract}

Maastricht Universitair Medisch Centrum (MUMC+), School for Public Health and Primary Care (CAPHRI), vakgroep Huisartsgeneeskunde, Postbus 616, 6200 MD Maastricht: R. Willemsen, huisarts-onderzoeker en kaderhuisarts hart- en vaatziekten; prof.dr. G.J. Dinant, huisarts. Hartcentrum, Onze Lieve Vrouwe Gasthuis, Amsterdam: prof.dr. F. Verheugt, cardioloog. Maastricht Universitair Medisch Centrum (MUMC+) en Cardiovascular Research Institute Maastricht (CARIM), Maastricht: prof.dr. H. ten Cate, internist. Medisch Centrum Haaglanden-Bronovo ziekenhuis, Den Haag: N. Weerkamp, neuroloog • Correspondentie: robert.willemsen@maastrichtuniversity.nl• Mogelijke belangenverstrengeling: niets aangegeven. verschillen tussen medicamenten gebruiken we telkens het number needed to treat (NNT), dat een objectiever beeld geeft dan relatieve risicoverschillen.

\section{BLOEDSTOLLING}

Geactiveerde trombocyten hechten onder invloed van tromboxane en adenosinedifosfaat aan elkaar en aan de vaatwand (plaatjesaggregatie). Daarnaast worden bij trombusvorming stollingsfactoren geactiveerd, zodat factor II (protrombine) wordt omgezet in trombine, en factor I (fibrinogeen) in fibrinedraden. Een stabiel stolsel bestaat dan ook uit trombocyten en fibrinedraden. De balans tussen pro- en antitrombotische mediatoren is individueel variabel. Zo hebben patiënten met een familiaire belasting voor hart- en vaatziekten in de regel een verhoogde trombogeniciteit. In een [webfiguur] bij dit artikel is de bloedstolling schematisch weergegeven (zie www.henw. org, rubriek Nascholing).

\section{Ziekte door bloedstolling}

Bij arteriële hart- en vaatziekten leidt atherosclerotische endotheelschade incidenteel tot stollingsactivatie met infarcering tot gevolg (myocardinfarct, herseninfarct). ${ }^{2}$ In andere gevallen leidt atherosclerose tot stabiele stenosering zonder stollingsactivatie, bijvoorbeeld bij stabiel kransslagaderlijden en perifeer arterieel vaatlijden. In het gehele arteriële vaatbed van deze patiënten bestaat er echter een verhoogd risico op atherosclerotische schade mét stollingsactivatie. ${ }^{2}$ Bij veneuze trombotische aandoeningen leidt een combinatie van veranderde bloedsamenstelling, vaatwandschade en een trage bloedstroom (trias van Virchow) tot trombusvorming. ${ }^{3}$

\section{De kern}

- Trombocytenaggregatie enerzijds en vorming van fibrinedraden via de stollingsfactoren anderzijds leiden tot een stabiel stolsel. Elk antitrombotisch medicament grijpt aan via een van deze twee systemen.

- De keuze voor de aard van de antitrombotische therapie is afhankelijk van de indicatie.

- Daarnaast moet men het individuele bloedingsrisico van de patiënt meewegen.

- Orale anticoagulantia zijn vooral van waarde bij een verhoogd veneus trombotisch risico, plaatjesremming bij een verhoogd arterieel trombotisch risico.

- Combinaties van twee verschillende soorten plaatjesremmers zijn in sommige situaties te prefereren boven monotherapie met één plaatjesremmer.

- Actueel onderzoek richt zich op de vraag bij welke patiënten een combinatie van drie verschillende antitrombotische middelen gezondheidswinst oplevert. 
Figuur Overzicht van de verschillende soorten antitrombotische medicamenten. Antitrombotische medicatie is de overkoepelende naam voor medicamenten die trombusvorming remmen. Deze groep bestaat uit plaatjesremmers enerzijds en orale antistolling (OAC) anderzijds.

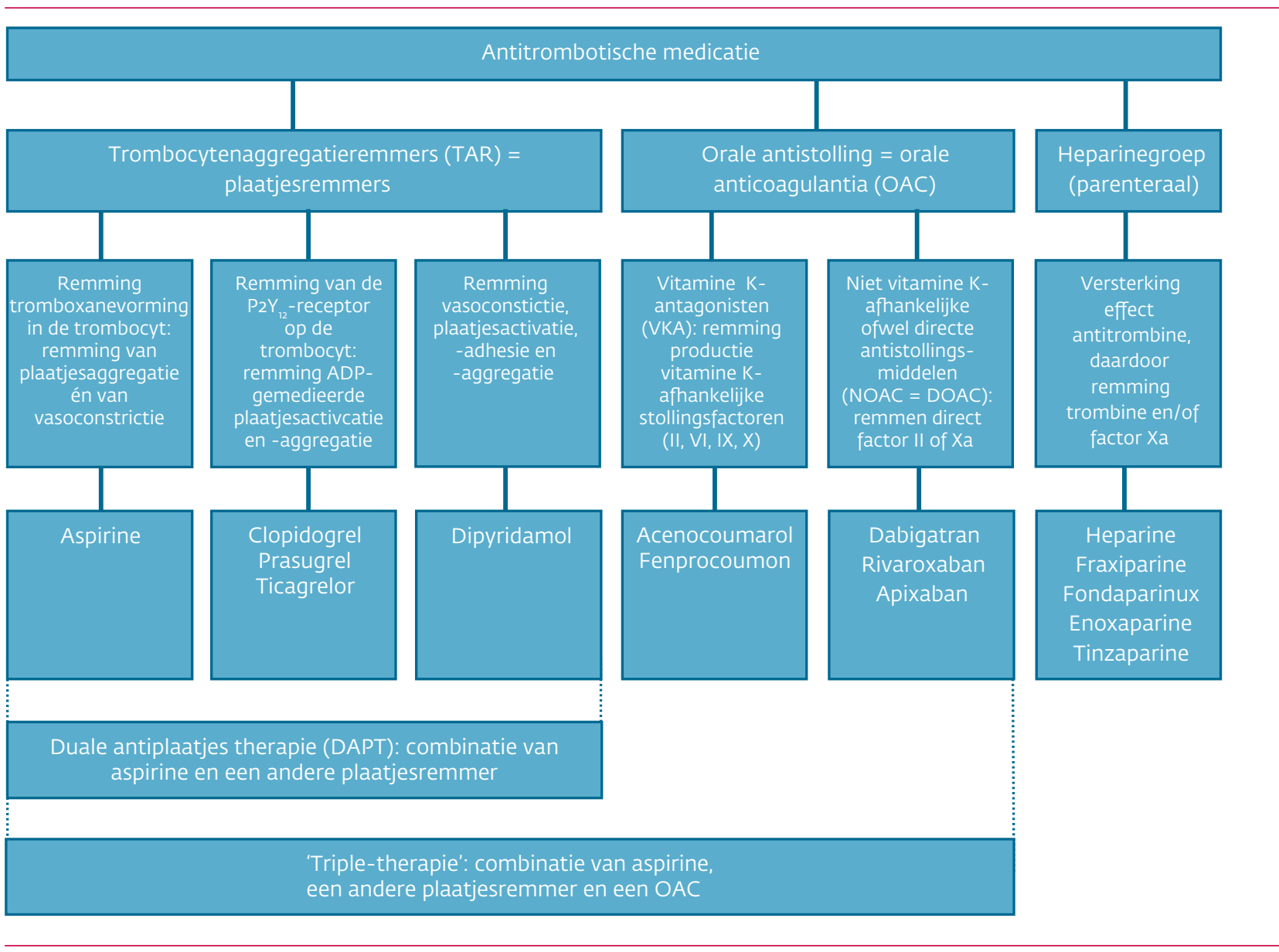

\section{Antitrombotische medicatie: plaatjesremmers of OAC}

Antitrombotische medicatie is de overkoepelende naam voor alle stollingsremmende medicatie ([figuur] en [webfiguur], zie www.henw.org, rubriek Nascholing). De niet geheel juiste term 'bloedverdunners' wordt hier ook voor gebruikt. Antitrombotische medicatie werkt ófwel door aan te grijpen op het systeem van de trombocyten (de groep van de plaatjesremmers of trombocytenaggregatieremmers) ófwel door aan te grijpen op het systeem van de stollingsfactoren (de groep van de orale antistollingsmiddelen of orale anticoagulantia, afgekort als $O A C$ ). Plaatjesremmers worden vooral ingezet bij een verhoogd risico op arteriële trombusvorming, zoals secundaire preventie van hart- en vaatziekten. OAC gebruikt men traditioneel bij veneuze stollingsproblemen (diepe veneuze trombose, longembolie, preventie van trombo-embolieën bij atriumfibrilleren). Heparineachtigen werken ook via de stollingsfactoren, maar bestaan niet in orale toedieningsvorm.

\section{Combinaties van middelen: DAPT, triple-therapie}

De term 'duale antiplaatjestherapie" (DAPT) verwijst naar een combinatie van aspirine en een andere plaatjesremmer. Omdat aspirine tromboxane remt en de andere plaatjesremmers

\section{Abstract}

Willemsen $R$, Dinant GJ, Verheugt F, Ten Cate H, Weerkamp N. Anticoagulation in 2016. Huisarts Wet 2016;59(7):312-8.

This article provides an overview of current antithrombotic therapy, a topic about which general practitioners often have questions. The pathophysiology of thrombus formation and the different types of currently available oral antithrombotic drugs are described, and then the preferred antithrombotic regimen for individual indications is discussed. Pathological thrombus formation is caused by either venous thrombosis (as is the case in pulmonary embolism) or arterial thrombosis (as is the case in myocardial infarction and stroke without atrial fibrillation). In both cases, antithrombotic medication is usually indicated. There are two main groups of oral antithrombotic drugs: antiplatelet drugs and anticoagulants. The former are usually indicated when there is a high risk of arterial thrombosis; for example, in stable coronary artery disease, peripheral artery disease, and stroke. In contrast, oral anticoagulants are usually indicated in the case of venous thrombosis or an increased risk thereof (atrial fibrillation, deep venous thrombosis, or pulmonary embolism). However, the preferred type of antithrombotic agent varies by indication. Although the risk of major bleeding should be taken into consideration, it is rarely an absolute contraindication for antithrombotic therapy. Occasionally combinations of different antithrombotic agents are indicated. For example, two different antiplatelet drugs maybe preferred instead of monotherapy in certain situations, such as after myocardial infarction, and two or three drugs can be used in patients with previous myocardial infarction and atrial fibrillation. 
Tabel Overzicht van de actuele antitrombotische behandelschema's per indicatie.

\begin{tabular}{|c|c|c|c|}
\hline \multicolumn{2}{|l|}{ Indicatie } & Voorkeursbehandeling & Opmerkingen \\
\hline \multicolumn{2}{|c|}{ Primaire preventie en diabetes mellitus type 2} & Géén antitrombotische therapie & $\begin{array}{l}\text { Enige reductie van optreden hart- en vaatziekten, maar } \\
\text { weegt niet op tegen bloedingsrisico }\end{array}$ \\
\hline \multicolumn{2}{|c|}{ Stabiele angina pectoris } & Aspirine (levenslang) & Bij overgevoeligheid aspirine: clopidogrel \\
\hline \multicolumn{2}{|c|}{ Perifeer arterieel vaatlijden } & Aspirine (levenslang) & Bij overgevoeligheid aspirine: clopidogrel \\
\hline \multicolumn{2}{|c|}{ Aneurysma aortae abdominalis } & Aspirine (levenslang) & $\begin{array}{l}\text { Niet goed onderzocht, algemeen advies: aspirine gezien } \\
\text { belangrijke correlatie aneurysma aortae abdominalis en } \\
\text { atherosclerose }\end{array}$ \\
\hline \multirow[t]{4}{*}{$\begin{array}{l}\text { Acuut coronair } \\
\text { syndroom }\end{array}$} & $\begin{array}{l}\text { met ST-elevaties en/of } \\
\text { stentplaatsing }\end{array}$ & & \\
\hline & & DAPT gedurende de eerste $9-12$ maanden & \\
\hline & $\begin{array}{l}\text { zonder ST-elevaties en/of } \\
\text { stentplaatsing }\end{array}$ & Aspirine (levenslang) & \\
\hline & & DAPT gedurende de eerste 12 maanden & \\
\hline \multirow[t]{2}{*}{ Herseninfarct/ TIA } & zonder atriumfibrilleren & $\begin{array}{l}\text { Eerste twee weken aspirine met dipyridamol } \\
\text { óf met clopidogrel. Daarna onderhoud: } \\
\text { clopidogrel monotherapie óf DAPT (te weten } \\
\text { aspirine en dipyridamol) }\end{array}$ & $\begin{array}{l}\text { Monotherapie met clopidogrel is goedkoper en leidt tot } \\
\text { minder hoofdpijn én minder bloedingscomplicaties, } \\
\text { maar wordt vooral door de neuroloog voorgeschreven. } \\
\text { In de NHG-Standaard wordt aspirine met dipyridamol } \\
\text { als eerste keus aangehouden. }\end{array}$ \\
\hline & met atriumfibrilleren & $\begin{array}{l}\text { Zie 'atriumfibrilleren met CHA2DS2-Vasc- } \\
\text { score > } 1 \text { ' }\end{array}$ & \\
\hline \multicolumn{2}{|c|}{ Diepe veneuze trombose } & OAC (VKA of NOAC) & $\begin{array}{l}\text { Keuze OAC: VKA en NOAC gelijkwaardig } \\
\text { Duur: } 3 \text { maanden bij directe aanleiding (bijvoorbeeld } \\
\text { postoperatief), gedurende } 6 \text { maanden bij idiopathische } \\
\text { diepe veneuze trombose }\end{array}$ \\
\hline \multicolumn{2}{|l|}{ Longembolie } & OAC (VKA of NOAC) & $\begin{array}{l}\text { Keuze OAC: VKA en NOAC gelijkwaardig } \\
\text { Duur: } 6 \text { maanden, levenslang bij recidief }\end{array}$ \\
\hline \multicolumn{2}{|c|}{$\begin{array}{l}\text { Atriumfibrilleren met CHA2DS2-Vasc-score } \\
\geq 1 \text { (ESC) of } \geq 2(\mathrm{NHG})\end{array}$} & OAC & $\begin{array}{l}\text { Bij CHA2DS2-Vasc-score } \leq 1 \text { géén aspirine, noch andere } \\
\text { antitrombotische therapie } \\
\text { ESC-richtlijn adviseert ook bij CHA2DS2-Vasc-score = } 1 \\
\text { OAC (met uitzondering van score van } 1 \text { door vrouwelijk } \\
\text { geslacht zonder andere risicofactoren), bij CHA2DS2- } \\
\text { Vasc-score = } 0 \text { géén antitrombotische therapie } \\
\text { Keuze OAC: NHG-Standaard voorkeur VKA, ESC-richtlijn } \\
\text { voorkeur NOAC }\end{array}$ \\
\hline \multicolumn{2}{|c|}{ Atriumfibrilleren én acuut coronair syndroom } & $\begin{array}{l}\text { Afhankelijk van comorbiditeit en bloedingsri- } \\
\text { sico individuele patiënt: triple-therapie }\end{array}$ & $\begin{array}{l}\text { Bewijs voor optimale strategie ontbreekt nog, } \\
\text { individuele afweging. } \\
\text { Triple-therapie of OAC combineren met } 1 \text { type } \\
\text { plaatjesremmer. } \\
\text { Bij te veel risico eventueel monotherapie met OAC }\end{array}$ \\
\hline
\end{tabular}

Afkortingen: DAPT = duale antiplaatjestherapie; OAC = orale anticoagulantia (= orale antistolling, omvat NOAC én VKA); NOAC = niet vitamine $\mathrm{K}$-afhankelijke (of 'nieuwe') orale anticoagulantia; VKA = vitamine $\mathrm{K}$-afhankelijke orale anticoagulantia

voornamelijk de adenosinedifosfaatreceptor ( $\mathrm{P}_{2} \mathrm{Y}_{12}$-receptor) remmen, wordt de trombocytenfunctie met DAPT sterker geremd dan met aspirine alleen. Triple-therapie is een combinatie van DAPT en een OAC.

\section{Indicaties}

Bij patiënten met een doorgemaakte hart- en vaatziekte reduceert aspirine de kans op een recidief met $25 \%$ (NNT is 73 patienten per jaar). ${ }^{4}$ Voor diverse indicaties is er een meerwaarde van aanvullende of andere antitrombotische therapie. We geven daarom een beknopt overzicht van het geïndiceerde antitrombotische regime per ziektebeeld [tabel].

\section{Primaire preventie en diabetes mellitus type 2}

Noch bij primaire preventie, noch bij patiënten met diabetes mellitus type 2 heeft antitrombotische medicatie een reducerend effect op het optreden van hart- en vaatziekten, dat opweegt tegen het hogere bloedingsrisico. In een grote metaanalyse over het effect van aspirine als primaire preventie, was het NNT om één cardiovasculair incident te voorkomen 720 per jaar. Eén op de 438 patiënten kreeg bij aspirinegebruik een bloeding en er was geen effect op de totale sterfte. ${ }^{5}$ Ook voor patiënten met diabetes mellitus type 2 zónder doorgemaakte hart- en vaatziekte heeft aspirine geen meerwaarde. ${ }^{6}$

Stabiele angina pectoris, perifeer arterieel vaatlijden en aneurysma aortae abdominalis

Patiënten met stabiele angina pectoris of perifeer arterieel vaatlijden hebben een verhoogd risico op cardiovasculaire incidenten.7,8 Stollingsactivatie door atherosclerotische vaat- 
wandschade treedt bij hen meestal niet op ter plaatse van de bekende stabiele stenoses, maar ter hoogte van instabiele atherosclerotische plaques elders in het vaatbed..$^{2,-11}$ De kans daarop bij stabiele angina pectoris is te reduceren met aspirine $(\mathrm{NNT}=12 \mathrm{o} / \mathrm{jaar}) .{ }^{12}$ Voor zover onderzocht lijkt er hierbij geen meerwaarde van een andere plaatjesremmer in plaats van aspirine, noch van DAPT. ${ }^{13-15}$ Bij patiënten met perifeer arterieel vaatlijden is aspirine eerste keus als secundaire preventie. Clopidogrel lijkt nog iets effectiever, maar heeft in de NHGStandaard niet de voorkeur boven aspirine. ${ }^{16,17}$ DAPT heeft op de lange termijn geen overtuigende meerwaarde. ${ }^{13,18}$ Omdat er een sterk verband bestaat tussen aneurysma aortae abdominalis en andere atherosclerotische ziekte raden de richtlijnen aspirine aan bij aneurysma aortae abdominalis, vooral in geval van cardiovasculaire comorbiditeit. ${ }^{17,19}$ Dit advies is onder voorbehoud, omdat goed onderzoek op dit vlak ontbreekt.

\section{Acuut coronair syndroom}

Het acuut coronair syndroom omvat myocardinfarct en instabiele angina pectoris. Instabiele angina pectoris komt steeds minder voor door de hoge sensitiviteit van moderne troponinetests. ${ }^{20} \mathrm{Na}$ een doorgemaakt myocardinfarct is het NNT om een tweede manifestatie van hart- en vaatziekte te voorkomen 63 patiënten per jaar voor aspirine, vergeleken met placebo. ${ }^{4}$ Bij overgevoeligheid dient men clopidogrel voor te schrijven. ${ }^{16}$ Zowel na een myocardinfarct met ST-elevaties als na stentplaatsing adviseren de ESC-richtlijnen gedurende negen tot twaalf maanden bovendien DAPT. ${ }^{21}$ Het NNT in dat eerste jaar is 32 , in vergelijking met aspirine alleen. ${ }^{22}$ Zowel een mogelijk langere behandeling met DAPT als de ideale combinatie van de diverse plaatjesremmers blijft onderwerp van actueel onderzoek. ${ }^{23-25}$

Ook bij een doorgemaakt myocardinfarct zonder ST-elevaties wordt gedurende het eerste jaar DAPT (aspirine met in dit geval bij voorkeur prasugrel of ticagrelor) geadviseerd vanwege een kleine meerwaarde. ${ }^{26-28} \mathrm{Na}$ het eerste jaar is monotherapie met aspirine effectief als secundaire preventie. ${ }^{4}$

\section{Herseninfarct/TIA zonder atriumfibrilleren}

Aspirine, gestart in de vroege fase na een herseninfarct, reduceert de kans op een snel recidief herseninfarct; het NNT is 79 om één geval van sterfte of afhankelijkheid binnen een half jaar na het eerste herseninfarct te voorkomen. ${ }^{29}$ Bovendien hangt op de langere termijn DAPT bestaande uit dipyridamol en aspirine samen met minder vasculaire sterfte, niet fataal herseninfarct en niet fataal myocardinfarct, vergeleken met aspirine monotherapie $\left(\mathrm{NNT}=100\right.$ per jaar).$^{30}$ Clopidogrel-monotherapie is recentelijk een goed alternatief gebleken voor de combinatie aspirine en dipyridamol..$^{31}$ Clopidogrel is even effectief, een factor 8 tot 10 goedkoper, geeft minder bloedingscomplicaties en leidt minder vaak tot hoofdpijn en discontinueren van de therapie. Neurologen schrijven clopidogrelmonotherapie op deze manier geregeld als eerste keus voor, maar deze behandeling heeft nog geen definitieve plek in de NHG-Standaard. Wanneer men voor clopidogrel kiest, luidt het advies om uitsluitend gedurende de eerste twee weken na het herseninfarct of de TIA nog wel DAPT (aspirine gecombineerd met clopidogrel óf dipyridamol) voor te schrijven. ${ }^{33,34}$

\section{Diepe veneuze trombose en longembolieën}

Bij diepe veneuze trombose of longembolie zijn OAC geïndiceerd. 'Nieuwe' ofwel 'niet vitamine K-afhankelijke orale anticoagulantia' (NOAC's) en de wijd ingeburgerde vitamine K-antagonisten (VKA) hebben een gelijkwaardig gunstig effect op morbiditeit en mortaliteit. ${ }^{35,36}$ Met sommige NOAC's zijn lagere bloedingsrisico's beschreven. ${ }^{37}$ Tot er een adequate instelling is bereikt, moet men een laagmoleculairgewichtheparine geven bij VKA en bij sommige NOAC's (dabigatran, edoxaban). Mogelijk is dabigatran (een NOAC) kosteneffectief voor de behandeling van diepe veneuze trombose, in vergelijking met VKA via de (Nederlandse) trombosedienst. ${ }^{38}$ Bij de behandeling van diepe veneuze trombose of longembolie is er in eerste instantie geen plaats voor plaatjesremmers. Wanneer men bij diepe veneuze trombose of longembolie ná de behandelfase met OAC (meestal drie tot zes maanden) een plaatjesremmer als onderhoudstherapie geeft, is er een lager recidiefrisico $(\mathrm{NNT}=42$ gedurende 1 jaar met aspirine vergeleken met placebo). ${ }^{39}$ Dit is bij een relatief klein aantal mensen onderzocht en de NHG-Standaard beveelt deze strategie daarom niet aan. ${ }^{40}$

\section{Atriumfibrilleren}

Patiënten met atriumfibrilleren hebben een verhoogd risico op een herseninfarct, ook wanneer zij een (nagenoeg) permanent sinusritme hebben. ${ }^{41-44}$ Dit risico is te schatten met behulp van de $\mathrm{CHA}_{2} \mathrm{DS}_{2}$-VASc-score. Het NNT om één herseninfarct te voorkomen bij atriumfibrilleren voor alle patiënten samen (ongeacht $\mathrm{CHA}_{2} \mathrm{DS}_{2}$-VASc-score) is 26 per jaar voor VKA vergeleken met placebo. ${ }^{45}$ Het effect van aspirine is inferieur door een minder sterk effect op het gecombineerd eindpunt van herseninfarct en hersenbloeding $(\mathrm{NNT}=74$ per jaar om één trombotisch of bloedig incident te voorkomen). Het NNT om één herseninfarct te voorkomen met OAC versus plaatjesremming is $71 .{ }^{45}$ Aspirine heeft bovendien, vooral bij ouderen, een gelijkwaardig risico op ernstige bloedingen als OAC. ${ }^{46,47}$ Ook DAPT is inferieur aan OAC bij atriumfibrilleren. ${ }^{48}$ Het routinematig gebruik van plaatjesremmers bij atriumfibrilleren voor preventie van een herseninfarct is dan ook obsoleet, tenzij er sprake is van intolerantie voor OAC.44,49,50

De NHG-Standaard adviseert om aan patiënten met een $\mathrm{CHA}_{2} \mathrm{DS}_{2}$-VASc-score $\geq 2$ OAC voor te schrijven. De NHG-Standaard geeft de voorkeur aan een VKA, in verband met de nog geringe ervaring met NOAC's bij oudere eerstelijnspatiënten en multimorbiditeit. ${ }^{49}$ In Nederland kan de huisarts volgens de huidige afspraken met de zorgverzekeraars een NOAC niet starten. De Europese huisartsenrichtlijn Herseninfarctpreventie bij atriumfibrilleren (EPCCS-richtlijn) stelt dat OAC bij een $\mathrm{CHA}_{2} \mathrm{DS}_{2}$-VASc-score $\geq 2$ absoluut en bij een score van 1 relatief geïndiceerd is. ${ }^{44}$ Deze richtlijn beschouwt VKA en NOAC's als gelijkwaardig. De voor- en nadelen van beide mid- 
delen zijn verschillend, maar niet doorslaggevend, tenzij er een duidelijke voorkeur van de patiënt is of sprake is van sterk wisselende INR-waarden onder VKA.

De Europese cardiologenrichtlijn (ESC-richtlijn) adviseert $\mathrm{OAC}$ zelfs al voor te schrijven bij een $\mathrm{CHA}_{2} \mathrm{DS}_{2}$-VASc-score $\geq$ $1 .{ }^{50} \mathrm{De}$ ESC-richtlijn spreekt een voorkeur uit voor NOAC boven VKA. Vooral vanuit de tweede lijn is er dus een toenemend aantal patiënten dat een NOAC krijgt. NOAC's hebben weliswaar als voordeel dat metingen van de INR niet nodig zijn, maar tóch verdient ook de patiënt op een NOAC intensieve opvolging om therapietrouw te waarborgen en complicaties te registreren, bijvoorbeeld via de trombosedienst. ${ }^{51}$ Patiënten met atriumfibrilleren bij kleplijden dienen altijd VKA te krijgen en geen NOAC. ${ }^{2}$

Vergeleken met patiënten die goed zijn ingesteld op VKA, neemt het aantal herseninfarcten bij atriumfibrilleren met NOAC's niet af. ${ }^{33}$ NOAC's leiden wel tot minder intracraniële bloedingen als complicatie, in vergelijking met VKA. Het NNT om één intracraniële bloeding te voorkomen met een NOAC versus een VKA is 500 per jaar.35 ${ }^{3 i j}$ ouderen ( $\geq 75$ jaar) is er wel sprake van een hoger aantal tractus digestivus-bloedingen bij NOAC-gebruik. ${ }^{54}$ Een ander onderzoek laat op alle leeftijden bij dabigatran-gebruik meer majeure, meer tractus digestivus-, maar minder intracraniële bloedingen zien. ${ }^{55}$ Anderzijds neemt in dit onderzoek het intracraniële bloedingsrisico bij VKA-gebruik toe bij patiënten vanaf 75 jaar. Op grond hiervan is geen eenduidig beleid bij ouderen te formuleren; de keuze voor een VKA of een NOAC blijft bij deze groep patiënten voorlopig een individuele afweging.

\section{MEERDERE AANDOENINGEN BIJ ÉÉN PATIËNT} Atriumfibrilleren en doorgemaakt acuut coronair syndroom Bij patiënten met atriumfibrilleren en daarnaast een doorgemaakt myocardinfarct met ST-elevaties of een stentplaatsing is er volgens de Europese cardiologenrichtlijn soms een indicatie voor triple-therapie: een combinatie van DAPT én antistolling. ${ }^{21}$ Maar deze richtlijn noemt triple-therapie ook een 'controversieel gebied' dat gekenmerkt wordt door kennislacunes. Bij ouderen heeft triple-therapie in elk geval geen meerwaarde boven DAPT als het gaat om de relevante uitkomstmaten, maar verhoogt het wel sterk het bloedingsrisico..$^{56}$ Actueel onderzoek richt zich op de vraag of een NOAC, gecombineerd met een $\mathrm{P}_{2} \mathrm{Y}_{12}$-receptorremmende plaatjesremmer - dus zónder aspirine - een effectief en relatief veilig behandelregime is. ${ }^{57}$

\section{Atriumfibrilleren en herseninfarct}

Zeker 20\% van de herseninfarcten is toe te schrijven aan atriumfibrilleren. Deze herseninfarcten verlopen ernstiger, met een hogere kans op blijvende ernstige schade..$^{8}$ Na een herseninfarct of TIA en daarbij atriumfibrilleren (in de voorgeschiedenis, dan wel ontdekt in de diagnostische fase na een herseninfarct/TIA) is de $\mathrm{CHA}_{2} \mathrm{DS}_{2}$-VASc-score minimaal 2 en dus is er een harde indicatie voor OAC.

\section{Acuut coronair syndroom en herseninfarct}

Optimale antitrombotische medicatie bij patiënten met een doorgemaakt herseninfarct én acuut coronair syndroom is weinig onderzocht. In deze gevallen - wanneer er geen sprake is van atriumfibrilleren - lijkt DAPT het meest opportuun, gezien de aangetoonde effecten op beide aandoeningen. Wanneer er wél eveneens sprake is van atriumfibrilleren kan men triple-therapie overwegen; zie hierboven onder het kopje 'Atriumfibrilleren en doorgemaakt acuut coronair syndroom'.

\section{Bloedingen en contra-indicaties}

VKA en plaatjesremmers scoren van oudsher hoog als het gaat om medicatiegerelateerde problemen op een spoedeisende hulp.59,60 Ook met de goed werkende Nederlandse trombosediensten is het risico op een belangrijke bloeding onder VKA nog altijd 2\% per jaar. ${ }^{61}$ Desondanks vormt ook bij oudere patiënten met atriumfibrilleren een valrisico geen contra-indicatie voor OAC, omdat het risico op herseninfarct het grootst is en het vallen niet leidt tot significant meer bloedingen. ${ }^{46,62}$ Hoewel men de ziektelast van niet-fatale bloedingen niet mag onderschatten, nemen patiënten die vaak toch voor lief als ze daarmee een herseninfarct kunnen voorkomen. ${ }^{63}$ Het risico op een bloeding bij een patiënt met atriumfibrilleren is uit te rekenen met behulp van de HAS-BLED-score (zie www.mdcalc. com/has-bled-score-for-major-bleeding-risk/). ${ }^{64}$

Een aantal factoren uit de HAS-BLED-score verhoogt óók - en vaak nog sterker - het risico op stolselvorming, zoals leeftijd en hypertensie. Zelfs bij een hoge HAS-BLED-score prevaleert daardoor in de regel het antitrombotische effect en is er dus een gunstig effect van OAC, vooral bij een $\mathrm{CHA}_{2} \mathrm{DS}_{2}$-VAScscore $\geq 2 .{ }^{65}$ De HAS-BLED-score kent geen afkappunt waarboven antitrombotische medicamenten gecontraïndiceerd zijn, maar is wél behulpzaam bij het vaststellen en corrigeren van reversibele factoren die bijdragen aan het bloedingsrisico (zoals hypertensie of NSAID-gebruik).44 Overigens vormt ook een doorgemaakte bloeding niet altijd een contra-indicatie voor (continueren van) antitrombotische therapie. De manier waarop men omgaat met de antitrombotische medicatie en bloedingsrisico's blijft een individuele afweging, afhankelijk van het trombotische risico.

DAPT en triple-therapie geven een hoger risico op bloeding dan behandeling met één antitrombotisch medicament. Grofweg geldt dat DAPT het bloedingsrisico verdubbelt ten opzichte van monotherapie met aspirine en dat triple-therapie het risico zelfs verdrievoudigt. ${ }^{66}$

\section{CONCLUSIE}

Antitrombotische medicatie beïnvloedt het delicate systeem van de bloedstolling.

Bij (verhoogd risico op) veneuze trombose (atriumfibrilleren, diepe veneuze trombose, longembolie, TIA of herseninfarct door atriumfibrilleren) heeft OAC (VKA of NOAC) de voorkeur. Bij (verhoogd risico op) arteriële trombose (TIA, herseninfarct niet door atriumfibrilleren, stabiele angina pectoris, doorgemaakt acuut coronair syndroom, perifeer arterieel 
vaatlijden, aneurysma aortae abdominalis) vormen (combinaties van) diverse plaatjesremmers de hoeksteen van de behandeling.

Bij patiënten met verhoogde veneuze én arteriële trombotische risico's is er in individuele gevallen plaats voor combinaties van plaatjesremming en OAC.

Ondanks heldere richtlijnen blijft de keuze voor een specifiek antitrombotisch regime per patiënt afhankelijk van het individuele trombotische risico enerzijds en het bloedingsrisico anderzijds.

\section{DANKBETUIGING}

Wij bedanken Guusje Bertholet voor de webillustratie.

\section{LITERATUUR}

1 Cals J, Willemsen R. Risicoreductie en number needed to treat. Huisarts Wet 2012;56:19.

2 Kooij A. Cardiovasculair risicomanagement. Houten: Prelum Uitgevers, 2012.

3 Chung I, Lip GY. 'Virchow's triad revisited: blood constituents'. Pathophysiol Haemost Thromb 2003;33:449-54.

4 Antithrombotic Trialists' Collaboration. Collaborative meta-analysis of randomised trials of antiplatelet therapy for prevention of death, myocar dial infarction, and stroke in high risk patients. BMJ 2002;324:71-86.

5 Seshasai SRK, Wijesuriya S, Sivakumaran R, Nethercott S, Erqou S, Sattar $\mathrm{N}$, et al. Effect of aspirin on vascular and nonvascular outcomes. Metaanalysis of randomized controlled trials. Arch Intern Med 2012;172:209 16.

6 Ogawa H, Nakayama M, Morimoto T, Uemura S, Kanauchi M, Doi N. Low dose aspirin for primary prevention of atherosclerotic events in patients with type 2 diabetes: a randomized controlled trial. JAMA 2008;3000:2134 41.

7 Daly CA, De Stavola B, Sendon JL, Tavazzi L, Boersma E, Clemens F, et al. Predicting prognosis in stable angina: results from the Euro heart survey of stable angina: prospective observational study. BMJ 2006;332:262-7.

8 Tendera M, Aboyans V, Bartelink ML, Baumgartner I, Clément D, Collet JP, et al. ESC Guidelines on the diagnosis and treatment of peripheral artery diseases: document covering atherosclerotic disease of extracranial carotid and vertebral, mesenteric, renal, upper and lower extremity arteries: the Task Force on the Diagnosis and Treatment of Peripheral Artery Diseases of the European Society of Cardiology (ESC). Eur Heart 2011;32:2851-906.

9 Stergiopoulos K, Brown DL. Initial coronary stent implantation with medical therapy vs medical therapy alone for stable coronary artery disease: meta-analysis of randomized controlled trials. Arch Intern Med 2012;172:312-9.

10 Bangalore S, Pursnani S, Kumar S, Bagos PG. Percutaneous coronary in tervention vs. optimal medical therapy for prevention of spontaneous myocardial infarction in subjects with stable ischemic heart disease. Circulation 2013;127:769-81.

11 Sedlis SP, Hartigan PM, Teo KK, Maron DJ, Spertus JA, Mancini GB, et al. Effect of PCI on long-term survival in patients with stable ischemic heart disease. N Engl J Med 2015;373:1937-46.

12 Juul-Möller S, Edvardsson N, Jahnmatz B, Rosén A, Sørensen S, Ömblus R Double-blind trial of aspirin in primary prevention of myocardial infarc tion in patients with stable chronic angina pectoris. Lancet 1992;340:14215 .

13 Bhatt DL, Fox KA, Hacke W, Berger PB, Black HR, Boden WE, et al. Clopidogrel and aspirin versus aspirin alone for the prevention of atherothrombotic events. N Eng J Med 2006;354:1706-17.

14 Roe MT, Armstrong PW, Fox KA, White HD, Prabhakaran D, Goodman SC, et al. Prasugrel versus clopidogrel for acute coronary syndromes without revascularization. N Eng J Med 2012;367:1297-309.

15 Montalescot G, Sechtem U, Achenbach S, Andreotti F, Arden C, Budaj A, et al. 2013 ESC guidelines on the management of stable coronary artery disease: the Task Force on the management of stable coronary artery disease of the European Society of Cardiology. Eur Heart J 2013;34:29493003.

16 CAPRIE Steering Committee. A randomised, blinded, trial of clopidogrel versus aspirin in patients at risk of ischaemic events (CAPRIE). Lancet 1996:348:1329-39.

17 NHG-Standaard Cardiovasculair risicomanagement. www.nhg.org.

18 Cacoub PP, Bhatt DL, Steg PG, Topol EJ, Creager MA. Patients with peripheral arterial disease in the CHARISMA trial. Eur Heart J 2009*30:192-201.

19 Erbel R, Aboyans V, Boileau C, Bossone E, Bartolomeo RD, Eggebrecht H, et al. 2014 ESC guidelines on the diagnosis and treatment of aortic diseases: document covering acute and chronic aortic diseases of the thoracic and abdominal aorta of the adult. The task force for the diagnosis and treatment of aortic diseases of the European Society of Cardiology (ESC). Eur Heart J 2014;35:2873-926.

20 Mueller C. Biomarkers and acute coronary syndromes: an update. Eur Heart J 2014;35:552-6.

21 Steg PG, James SK, Atar D, Badano LP, Blömstrom-Lundqvist C, Borger MA, et al. ESC Guidelines for the management of acute myocardial infarction in patients presenting with ST-segment elevation: The Task Force on the management of ST-segment elevation acute myocardial infarction of the European Society of Cardiology (ESC). Eur Heart J 2012;33:2569-619.

22 Ten Berg JM, Plokker HWT, Verheugt FWA. Antiplatelet and anticoagulant therapy in elective percutaneous coronary intervention. Curr Control Trials Cardiovasc Med 2001;2:129-40.

23 Udell JA, Bonaca MP, Collet JP, Lincoff AM, Kereiakes DJ, Costa F, et al. Long-term dual antiplatelet therapy for secondary prevention of cardiovascular events in the subgroup of patients with previous myocardial infarction: a collaborative meta-analysis of randomized trials. Eur Heart J 2016:37:390-9.

24 Bonacaa MP, Bhatt DL, Cohen M, Steg PG, Storey RF, Jensen EC, et al. Longterm use of ticagrelor in patients with prior myocardial infarction. $\mathrm{N}$ Engl J Med 2015;372:1791-1800.

25 Magnani G, Storey F, Steg G, Bhatt DL, Cohen M, Kuder J, et al. Efficacy and safety of ticagrelor for long-term secondary prevention of atherothrombotic events in relation to renal function: insights from the PEGASUS-TIMI 54 trial. Eur Heart J 2016;37:400-8.

26 Wallentin L, Becker RC, Budaj A, Cannon CP, Emanuelsson H, Held C, et al. Ticagrelor versus clopidogrel in patients with acute coronary syndromes. N Engl J Med 2009;361:1045-57.

27 Wiviott SD, Braunwald E, McCabe CH, Montalescot G, Ruzyllo W, Gottlieb $S$, et al. Prasugrel versus clopidogrel in patients with acute coronary syndromes. N Engl J Med 2007;357:2001-15

28 Roffi M, Patrono C, Collet JP Mueller C, Valgimigli M, Andreotti F, et al. 2015 ESC Guidelines for the management of acute coronary syndromes in patients presenting without persistent ST-segment elevation: The Task Force for the management of acute coronary syndromes (ACS) in patients presenting without persistent ST-segment elevation of the European Society of Cardiology (ESC). Eur Heart J 2015;DOI:10.1093/eurheartj/ehv320.

29 Sandercock PA, Counsell C, Gubitz GJ, Tseng MC. Antiplatelet therapy for acute ischemic stroke. Cochrane Database Syst Rev 2008;CD000029.

30 Halkes PH, Van Gijn J, Kappelle LJ, Koudstaal PJ, Algra A. Aspirin plus dipyridamole versus aspirin alone after cerebral ischaemia of arterial origin (ESPRIT): randomised controlled trial. Lancet 2006;367:1665-73.

31 Sacco RL, Diener HC, Yusuf S, Cotton D, Ounpuu S, Lawton WA, et al. Aspirin and extended-release dipyridamole versus clopidogrel for recurrent stroke. N Engl J Med 2008;359:1238-51.

32 Zinkstok SM, Zuurbier SM, Roos YBWEM. Plaatjesremming na een TIA of herseninfarct: de stand van zaken. Tijdschr Neurol Neurochir 2015;116:26 34.

33 Verheugt FW, Geersing GJ, Van Kapelle LJ. Toegevoegde waarde van clopidogrel in cardiologie en neurologie. Ned Tijdschr Geneeskd 2014;158:A7609.

34 Diener HC, Bogousslavsky J, Brass LM, Cimminiello C, Csiba L, Kaste M, et al. Aspirin and clopidogrel compared with clopidogrel alone after recent ischaemic stroke or transient ischaemic attack in high-risk patients (MATCH): randomised, double-blind, placebo-controlled trial. Lancet 2004;364:331-7.

35 Adam SS, McDuffie JR, Ortel TL, Williams JW jr. Comparative effectiveness of warfarin and new oral anticoagulants for the management of atrial fibrillation and venous thromboembolism: a systematic review. Ann Intern Med 2012;157:796-807.

36 Konstantinides SV, Torbicki A, Agnelli G, Danchin N, Fitzmaurice D, Galie $\mathrm{N}$, et al. (The Task Force for the Diagnosis and Management of Acute Pulmonary Embolism of the European Society of Cardiology (ESC) Endorsed by the European Respiratory Society (ERS)). 2014 ESC Guidelines on the diagnosis and management of acute pulmonary embolism. Eur Heart J 2014;35:3033-80

37 Van der Hulle T, Kooiman J, Den Exter PL, Dekkers OM, Klok FA, Huisman MV. Effectiveness and safety of novel oral anticoagulants as compared with vitamin $\mathrm{K}$ antagonists in the treatment of acute symptomatic venous thromboembolism: a systematic review and meta-analysis. J Thromb Haemost 2014;12:320-8.

38 Van Leent MW, Stevanović J, Jansman FG, Beinema MJ, Brouwers JR, Postma MJ. Cost-effectiveness of dabigatran compared to vitamin-K antagonists for the treatment of deep venous thrombosis in The Netherlands using real-world data. PloS One 2015 Aug 4;10:e0135054.

39 Simes J, Becattini C, Agnelli G, Eikelboom JW, Kirby AC, Mister R, et al. Aspirin for the prevention of recurrent venous thromboembolism: the IN SPIRE collaboration. Circulation 2014;130:1062-71.

40 NHG-Standaard Diepe veneuze trombose en longembolie. www.nhg.org.

41 Wolf PA, Abbott RD, Kannel WB. Atrial fibrillation as an independent risk factor for stroke: the Framingham Study. Stroke 1991;22:983-8.

42 Wyse DG, Waldo AL, DiMarco JP, Domanski MJ, Rosenberg Y, Schron EB, et al. A comparison of rate control and rhythm control in patients with atrial fibrillation. N Engl J Med 2002;347:1825-33.

43 Van Gelder IC, Hagens VE, Bosker HA, Kingma JH, Kamp O, Kingma T, et al. 
A comparison of rate control and rhythm control in patients with recurrent persistent atrial fibrillation. N Engl J Med 2002;347:1834-40.

44 Hobbs FR, Taylor CJ, Jan Geersing G, Rutten FH, Brouwer JR; on behalf of the European Primary Care Cardiovascular Society (EPCCS) SPAF working group. European Primary Care Cardiovascular Society (EPCCS) consensus guidance on stroke prevention in atrial fibrillation (SPAF) in primary care. Eur J Prev Cardiol 2016;23:460-73.

45 Hart RG, Pearce LA, Aguilar MI. Meta-analysis: antithrombotic therapy to prevent stroke in patients who have nonvalvular atrial fibrillation. Ann Intern Med 2007;146:857-67.

46 Mant J, Hobbs FD, Fletcher K, Roalfe A, Fitzmaurice D, Lip GY, et al. Warfarin versus aspirin for stroke prevention in an elderly community population with atrial fibrillation (the Birmingham Atrial Fibrillation of the Aged Study, BAFTA): a randomised controlled clinical trial. Lancet 2007;370:493-503.

47 Hobbs FD, Roalfe AK, Lip GY, Fletcher K, Fitzmaurice DA, Mant J, et al. Performance of stroke risk scores in older people with atrial fibrillation not taking warfarin: comparative cohort study from BAFTA trial. BMJ 2011;342:d3653.

48 Diener HC, Lowenthal A. Antiplatelet therapy to prevent stroke: risk of brain hemorrhage and efficacy in atrial fibrillation. J Neurol Sci 1997;153:112.

49 NHG-Standaard Atriumfibrilleren. www.nhg.org.

50 Camm AJ, Lip GY, De Caterina R, Savelieva I, Atar D, Hohnloser SH, et al. 2012 focused update of the ESC guidelines for the management of atrial fibrillation: an update of the 2010 ESC guidelines for the management of atrial fibrillation. Developed with the special contribution of the European Heart Rhythm Association. Eur Heart J 2012;33:2719-47.

51 Ten Cate $\mathrm{H}$. Trombosediensten blijven van waarde. Med Contact 2015;42:2002-3.

52 Eikelboom JW, Connolly SJ, Brueckmann M, Granger CB, Kappetein AP, Mack MJ, et al. Dabigatran versus warfarin in patients with mechanical heart valves. N Engl J Med 2013;369:1206-14.

53 Wallentin L Yusuf S, Ezekowitz MD, ALings M, Flather M, Franzosi MC, Pais P, et al. Efficacy and safety of dabigatran compared with warfarin at different levels of international normalised ratio control for stroke prevention in atrial fibrillation: an analysis of the RE-LY trial. Lancet 2010;376:975-83.

54 Abraham NS, Singh S, Alexander GC, Heien H, Haas LR, Crown W, et al. Comparative risk of gastrointestinal bleeding with dabigatran, rivaroxa- ban, and warfarin: population based cohort study. BMJ 2015;350:h1857.

55 Hernandez I, Baik SH, Piñera A, Zhang Y. Risk of bleeding with dabigatran in atrial fibrillation. JAMA Intern Med 2015;175:18-24.

56 Hess CN, Peterson ED, Peng SA, de Lemos JA, Fosbol EL, Thomas L, et al. Use and outcomes of triple therapy among older patients with acute myocar dial infarction and atrial fibrillation. J Am Coll Cardiol 2015;66:616-27.

57 Verheugt FW. Triple therapy for percutaneous coronary intervention in atrial fibrillation: standard of care, or a nightmare soon to end? J Thromb Haemost 2015; Suppl 1:S332-5.

58 Lin HJ, Wolf PA, Kelly-Hayes M, Beiser AS, Kase CS, Benjamin EJ, et al. Stroke severity in atrial fibrillation. The Framingham study. Stroke 1996;27:1760-4

59 Budnitz DS, Shebab N, Kegler SR, Richards CL. Medication use leading to emergency department visits for adverse drug events in older adults. Ann Intern Med 2007:147:755-65.

60 Ruiter R, Visser LE, Rodenburg EM, Trifirò G, Ziere G, Stricker BH. Ziekenhuisopnames door geneesmiddelbijwerkingen bij 55-plussers in Nederland. Ned Tijdschr Geneeskd 2012;156:A5182.

61 Van der Meer FJ, Rosendaal FR, Vandenbroucke JP, Briet E. Bleeding complications in oral anticoagulant therapy. An analysis of risk factors. Arch Intern Med 1993;153:1557-62.

62 Donze J, Clair G, Hug B, Rodondi N, Waeber C, Cornuz J, et al. Risk of falls and major bleeds in patients on oral anticoagulation therapy. Am J Med 2012;125:773-8

63 Devereaux PJ, Anderson DR, Gardner MJ, Putnam W, Flowerdew GJ, Brownell BF, et al. Differences between perspectives of physicians and patients on anticoagulation in patients with atrial fibrillation: observational study. BMJ 2001;323:1218-22.

64 Pisters R, Lane DA, Nieuwlaat R, De Vos CB, Crijns HJ, Lip GY. A novel userfriendly score (HAS-BLED) to assess 1-year risk of major bleeding in patients with atrial fibrillation: the Euro Heart Survey. Chest 2010;138:1093 100.

65 Olesen JB, Lip GY, Hansen PR, Lindhardsen J, Ahlehoff O, Andersson C, et al. Bleeding risk in 'real world' patients with atrial fibrillation: comparison of two established bleeding prediction schemes in a nationwide cohort. J Thromb Haemost 2011;9:1460-7.

66 Hansen ML, Sørensen R, Clausen MT, Fog-Petersen ML, Raunsø J, Gadsbøll $\mathrm{N}$, et al. Risk of bleeding with single, dual or triple therapy with warfarin, aspirin, and clopidogrel in patients with atrial fibrillation. Arch Intern Med 2010;170:1433-41.

Robert Willemsen, Karen Konings

\section{ECG-casus: acute inspanningsintolerantie}

\author{
Casus \\ Patiënt: \\ Man, 74 jaar, vitaa \\ Voorgeschiedenis: \\ Hypertensie, DM2 \\ Medicatie: \\ Quinapril/hydrochloorthiazide $1 \times 20 / 12,5$ \\ $\mathrm{mg}$, metformine $3 \times 500 \mathrm{mg}$, simvasta- \\ tine: $1 \times 40 \mathrm{mg}$ \\ Anamnese: \\ Patiënt maakt dagelijks een wandeling \\ van ongeveer $4,5 \mathrm{~km}$. De laatste weken \\ is hij steeds minder gaan lopen vanwege \\ kortademigheid. Hij weet dit aan een \\ griepje maar nu kan hij helemaal niet \\ meer wandelen. Na enkele meters voelt \\ hij zich doodmoe. \\ Lichamelijk onderzoek: \\ Tensie 134/88 mmHg. Pols 52/min irregu- \\ lair. Hart: heldere harttonen, geen souf- \\ fles. Longen: vesiculair ademgeruis, geen \\ bijgeluiden. Extremiteiten: geen oedeem \\ Verder geen afwijkingen.
}

ECG

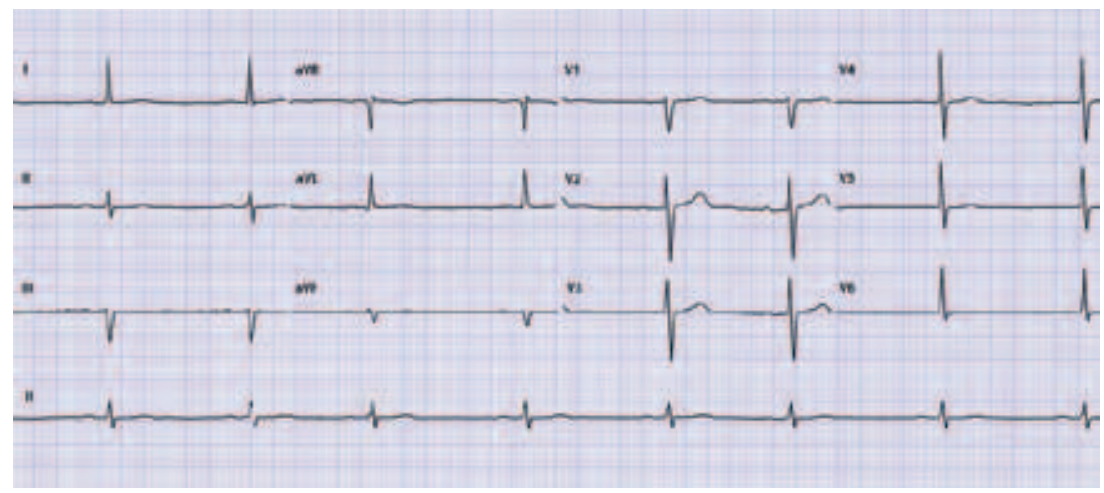

OPGAVE

Beschrijf het ECG systematisch volgens ECG-10 $10^{+}$en stel de diagnose in de conclusie. Wilt u ook een beleidsvoorstel doen?
Het juiste antwoord vindt $u$ op www. henw.org/oplossingcasus.

Konings KTS, Willemsen RTA. ECG 10+: Systematisch ECG's beoordelen. Huisarts Wet 2016;59:166 70. 